\title{
Generalized Manna Sandpile Model with Height Restrictions
}

\author{
Wellington Gomes Dantas and Jürgen F. Stilck \\ Instituto de Física, Universidade Federal Fluminense, Av. Litorânea s/n, 24210-340 - Niterói, RJ, Brazil
}

Received on 30 September, 2005

\begin{abstract}
Sandpile models with conserved number of particles (also called fixed energy sandpiles) may undergo phase transitions between active and absorbing states. We generalize the Manna sandpile model with fixed number of particles, introducing a parameter $-1 \leq \lambda \leq 1$ related to the toppling of particles from active sites to its first neighbors. In particular, we discuss a model with height restrictions, allowing for at most two particles on a site. Sites with double occupancy are active, and their particles may be transfered to first neighbor sites, if the height restriction do allow the change. For $\lambda=0$ each one of the two particles is independently assigned to one of the two first neighbors and the original stochastic sandpile model is recovered. For $\lambda=1$ exactly one particle will be placed on each first neighbor and thus a deterministic (BTW) sandpile model is obtained. When $\lambda=-1$ two particles are moved to one of the first neighbors, and this implies that the density of active sites is conserved in the evolution of the system, and no phase transition is observed. Through simulations of the stationary state, we estimate the critical density of particles and the critical exponents as functions of $\lambda$.
\end{abstract}

Keywords: Phase transitions; Absorbing states; Universality class; Sandpile model

\section{INTRODUCTION}

Problems related to phase transitions between active and absorbing states have attracted much interest in recent years [1]. Although these transitions occur away from thermodynamic equilibrium, since the presence of absorbing states prevents detailed balance to be satisfied in the dynamical evolution, the theoretical framework developed for equilibrium phase transition may in fact be applied to these systems, and concepts such as scaling and universality are relevant for nonequilibrium phase transitions as well. It is then of interest to identify the universality classes in these systems. Many of the stochastic models with absorbing states which show a phase transition belong to the directed percolation (DP) universality class [2], including the much studied contact process (CP) [3]. The so called DP conjecture [4], confirmed so far through many examples, stated originally that models with a scalar order parameter exhibiting phase transitions between an active state to a single absorbing state and without additional conservation laws should belong to this universality class. However it seems that models with multiple or even infinite absorbing states (without simmetries connecting them) are also included in this class $[5,6]$.In models for sandpiles the number of particles is conserved and an infinite number of absorbing states is present, and thus they are potential candidates to belong to a non-DP universality class. Careful simulations of the unrestricted Manna sandpile model [7] and the model with height restrictions [8] lead to exponents at variance with the DP value.

The Manna sandpile model was originally proposed as a stochastic model for SOC, with slow addition of sand and avalanches which lead to abrupt loss of sand [9]. The scaling behavior in the SOC regime was later recognized to be associated to an absorbing state phase transition in the corresponding model without addition or loss of particles (grains of sand), the so called fixed energy sandpiles (FES) [10]. In this model, a $d$-dimensional lattice of size $L^{d}$ is occupied by $N$ particles. A configuration of the lattice is specified fixing the number of particles $z_{i}$ at each site $i$, where $z_{i}$ may be any nonnegative integer number. Sites with $z_{i} \geq 2$ are active, and an active site may loose two particles to its first neighbors, with a unitary toppling rate. The two particles move to randomly and independently chosen sites among the first neighbors of site $i$. Any state in which no site has two or more particles is an absorbing state, and in the thermodynamic limit the number of absorbing states is infinite, as long as the density of particle $\zeta=N / L^{d}$ is smaller than unity. Numerical simulations [7] in fact show that this model in one dimension undergoes a continuous phase transition at a critical density $\zeta_{c}=0.9488$ with critical exponents which are different from the DP values.

In this paper we study a variation of the original model where the occupancy numbers are restricted to $z_{i} \leq 2$. Each move of a particle is accepted only if this constraint is not violated at the destination site. This additional restriction in the allowed configurations leads to simplifications in mean-field calculations and in simulations. Numerical simulations for the one-dimensional restricted model lead to a slightly lower critical density $\zeta_{c}=0.92965$, while the exponents are compatible with the ones found for the unrestricted model [8]. We generalize this restricted model by introducing a parameter whose value is related to the choice of the destination sites of the two toppling particles. The restricted Manna model corresponds to $\lambda=0$ and a model where exactly one particle is sent to each first neighbor of the active site (in one dimension) is recovered for $\lambda=1$. We call this latter case the BTW model, since it corresponds to the original Bak, Tang and Wiesenfeld model [11] with conserved number of particles. In particular, we are interested in investigating the phase transition in the model as the parameter $\lambda$ is changed.

In the section II we define the model in more details. The numerical simulations which were performed are described in section III, as well as the results they furnished. Conclusions may be found in section IV. 


\section{DEFINITION OF THE MODEL}

We consider $N$ particles located on the $L$ sites of a onedimensional lattice with periodic boundary conditions in such a way that each site $i$ is occupied by $z_{i}=0,1$ or 2 particles. Sites with two particles are active and the control parameter of the model is the density of particles $\zeta=N / L$. The update of the configuration is started by randomly choosing one of the $N_{A}$ active site of the lattice. The following updates of the configuration may then occur:

1. With a probability $(1+\lambda) / 2$ each first neighbor receives exactly one of the particles from the active site, as may be seen in Fig. 1.

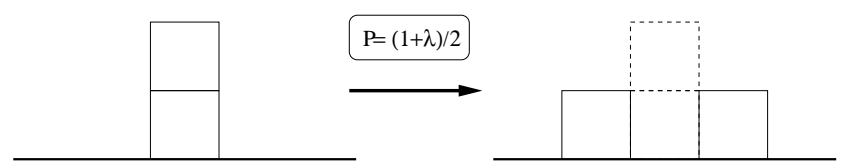

FIG. 1: The two particles of the active sites are moved to different first neighbor sites.

If one of the first neighbors is active, the particle which was chosen to be sent to this site remains on the original site. If both first neighbor sites are active, no particle movement occurs. This assures that the restriction on the occupancy numbers is always satisfied.

2 . With a probability $(1-\lambda) / 2$, one of the two first neighbors is chosen with probability $1 / 2$ and both particles are moved to this site. If the destination site is already occupied by one particle, only one of the particles originating from the active site is moved. If the destination site is active, no movement is done. An example of such a transition is shown in Fig. 2.

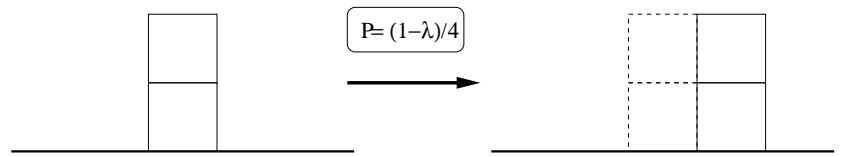

FIG. 2: Both particles from the active site are moved to the same first neighbor site.

One iteration as described above corresponds to a time increment of $1 / N_{A}$. After each iteration, the list of active sites is updated. In the restricted model the density of particles obeys $\zeta \leq 2$, and when $\zeta=2$ an additional absorbing state is reached. In the unrestricted model there is no such upper limit for $\zeta$ and no spurious absorbing state with unitary density of active sites exists. However, since the phase transition in the models occurs at particle densities below unity, this details do not bother us here. The fact that the restriction on the occupancy numbers implies a reduction of only about $2 \%$ in the critical density of the model reveals that for the unrestricted model in the supercritical region close to the phase transition the fraction of sites with occupancy larger than 2 should be very small.

The parameter $\lambda$ is restricted to the interval $[-1,1]$. In the upper limit of this interval, the toppling of particles is deterministic and the model corresponds to a BTW sandpile with conserved number of particles. In the lower limit the number of active sites is a conserved quantity in the time evolution of the model, with active sites diffusing on the lattice. The conservation of the number of active sites in this limit is a consequence of the occupancy numbers restriction and does not happen in the unrestricted model.

\section{SIMULATIONS AND RESULTS}

We realized simulations to find the critical properties of the one-dimensional model with height restrictions. The initial condition, for a given value of $\lambda$ and $\zeta=N / L$, is a uniform and uncorrelated distribution of the $N$ particles on the $L$ sites of the lattice, respecting the restrictions. We studied lattice sizes $L$ between 100 and 2000, performing $N_{r}$ repetitions with times up to $t_{\max }$ for a certain range of densities and each value of $\lambda$. In our simulations $t_{\max }$ was in the interval $\left[4 \times 10^{4}, 2 \times 10^{7}\right]$ and $N_{r}=2000$. Since in finite systems with absorbing states the only stationary states are the absorbing states themselves, to study the transition we are interested in the quasi-stationary states of the model. Usually, the simulational determination of this state is hindered by the presence of the absorbing states, since considerable fluctuations will be found in the quantities estimated in the simulations, particularly for particle densities close to the critical value. To avoid these drawbacks and increase the precision of the estimates of the stationary state we used the prescription for simulation of quasi-stationary states proposed recently by de Oliveira and Dickman [12].

In the simulations, the time evolution of the density of active sites $\rho_{a}$ is obtained, one example being shown in Fig. 3. A stationary value of this density may then be estimated for given values of $\zeta$ and $\lambda$ calculating the mean value of the last 2000 points in such simulations.

For each value of the lattice size $L$, realizing simulations for a range of particle densities $\zeta$, we obtain curves for the order parameter such as the one depicted in Fig. 4. It should be mentioned that for each lattice size the particle density may assume only a discrete set of values, but it seems reasonable to interpolate between those values, assuming that $\rho_{a}$ varies continuously with $\zeta$.

The estimated values of the order parameter as a function of the particle density $\zeta$ may then be analyzed considering the following scaling relations:

$$
\begin{aligned}
\rho_{a}\left(\zeta_{c}, L\right) & \sim L^{-\beta / v_{\perp}}, \\
\rho_{a}(\zeta) & \sim\left(\zeta-\zeta_{c}\right)^{\beta} .
\end{aligned}
$$

Here $\rho_{a}(\zeta)$ denotes the thermodynamic limit $L \rightarrow \infty$ of $\rho_{a}(\zeta, L)$. These scaling relations provide estimates for the critical exponents $\beta$ and $v_{\perp}$, as well as for the critical particle density $\zeta_{c}$. To estimate the critical particle density and the order parameter exponent $\beta$ we proceed as follows: for a 


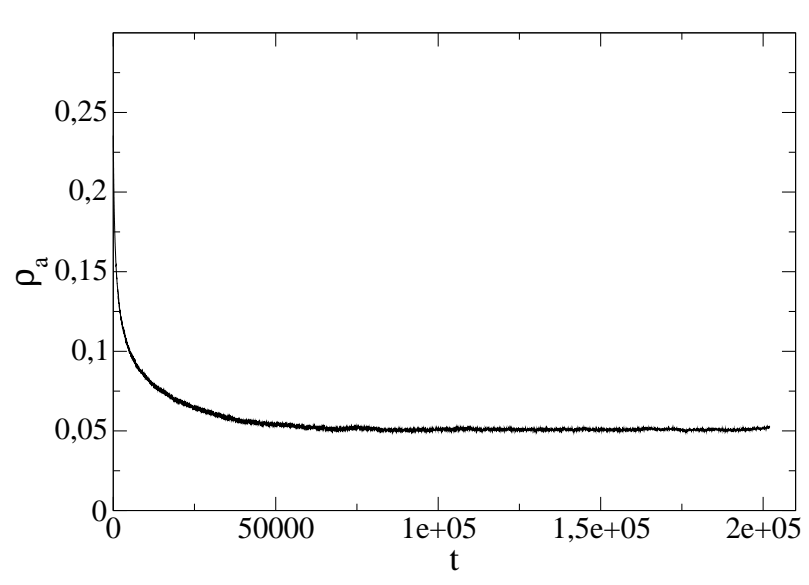

FIG. 3: Example of a simulation of the time evolution of the density of active sites. In this case $L=100, \lambda=0$, and $\zeta=0.92$.

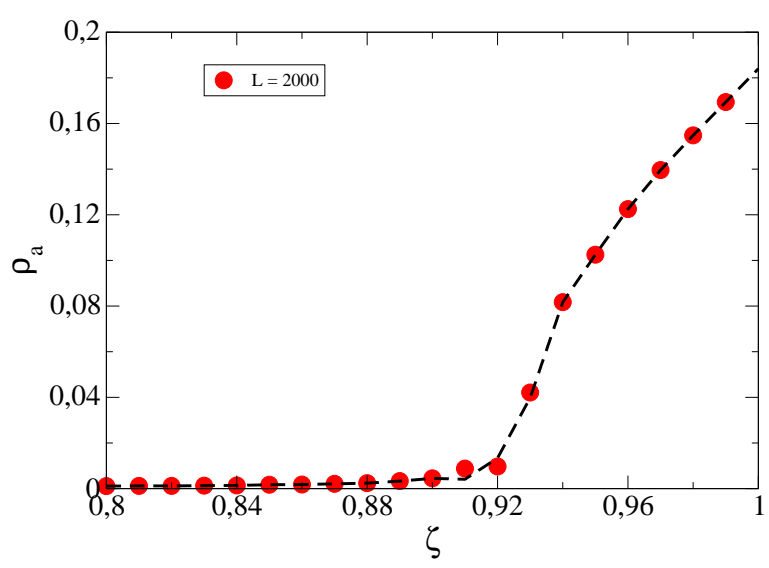

FIG. 4: Estimated values of the order parameter $\rho_{a}$ as a function of the particle density $\zeta$, obtained for $L=2000$ and $\lambda=0$. The dashed line is a guide for the eye.

given value of $L$ we choose a critical value $\zeta_{c}^{(L)}$ which maximizes the correlation in a linear approximation for the function $\ln \rho_{a}=b+\beta^{(L)} \ln \left[\zeta-\zeta_{c}^{(L)}\right]$, with $\zeta>\zeta_{c}^{(L)}$. Once obtaining estimates for $\beta^{(L)}$ and $\zeta_{c}^{(L)}$ for different values of $L$, they may be extrapolated to the limit $L \rightarrow \infty$. The ratio $\beta / v_{\perp}$ may be estimated directly through the relation $\ln \rho_{a}=c+\beta / v_{\perp} \ln L$, using values for $\rho_{a}$ at the estimated critical particle density.

Using the procedure described above, we obtained estimates for the case $\lambda=0$, as may be seen in Fig. 5. We then realized that the estimates for $\beta$ may be improved excluding the smaller sizes in the extrapolation to the thermodynamic limit. A better result for the ratio $\beta / v_{\perp}$ is found if we apply the scaling correction proposed in [12] for the simulation of the quasi-stationary state of the contact process. It seems that the algorithm proposed in this reference for simulations of the quasi-stationary state and used in our work implies a scaling correction which is characteristic of the model. With these corrections, our estimates are very close to the ones in reference [8]. The extrapolations with the scaling correction may be seen in Fig. 6 .
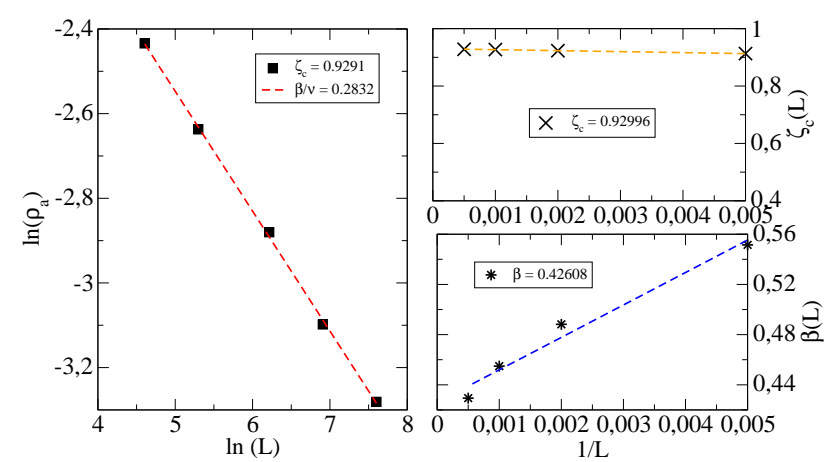

FIG. 5: Extrapolation without the scaling correction for $\lambda=0$. The estimates are: $\zeta_{c}=0.92996, \beta / v_{\perp}=0.2832$ and $\beta=0.42608$.

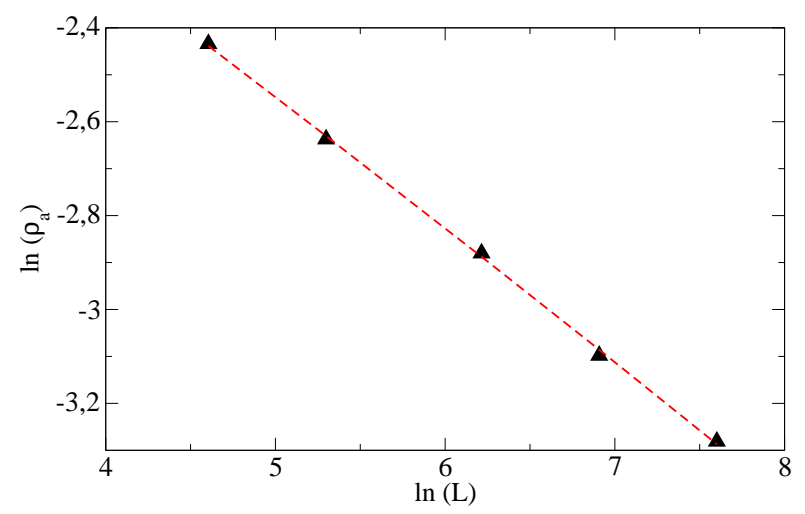

FIG. 6: Extrapolations with the scaling correction for $\lambda=0$. Corrected values for the estimates: $\zeta_{c}=0.92996, \beta / v_{\perp}=0.247$ and $\beta=0.41275$.

Repeating this strategy for different values of $\lambda$, we finally may obtain the phase diagram of the model, as well as study the values of the estimates for the critical exponents as functions of this parameter. In Fig. 7 the phase diagram is shown. Besides the points for the critical line which emerge from the simulations, the result of a 2-site mean field approximation for the model may also be seen in the figure. Details of these calculations will be given elsewhere. The curve which results from the mean-field calculations is always below the results of the simulations, confirming that when the correlations are ignored the size of the active region in the phase diagram is overestimated. As $\lambda$ approaches the limiting value -1 , the results of the simulations get closer to the ones in the mean-field approximation, which leads to $\zeta_{c}=1 / 2$ in this limit, even in a one-site approximation. This seems to be reasonable, since 
in this limit the evolution of the model is dominated by the diffusion of active sites, and this leads to a mean-field like behavior. This argument applies only in the limit of $\lambda$ slightly above -1 , since as already noticed above when $\lambda=-1, \rho_{a}(t)$ is constant and no phase transition is observed.

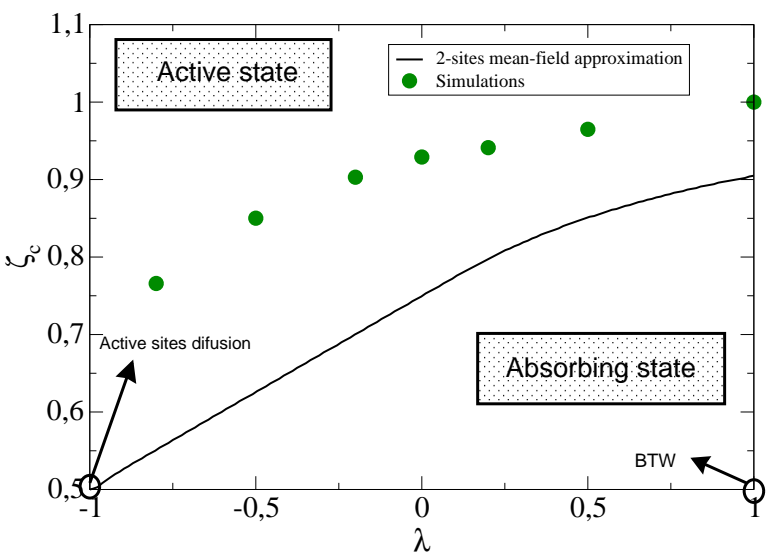

FIG. 7: Phase diagram of the Manna model with height restriction parametrized by $\lambda$.

In Fig. 8 we notice that the estimates for the critical exponents show a rather strong dependence of the parameter $\lambda$, which may be due to a crossover between universality classes in the limits $\lambda \rightarrow-1$, where mean-field behavior is expected, and $\lambda \rightarrow 1$, the conservative BTW model. In this latter limit, our simulations indicate a first-order transition at $\zeta=1$, as may be seen in Fig. 9.

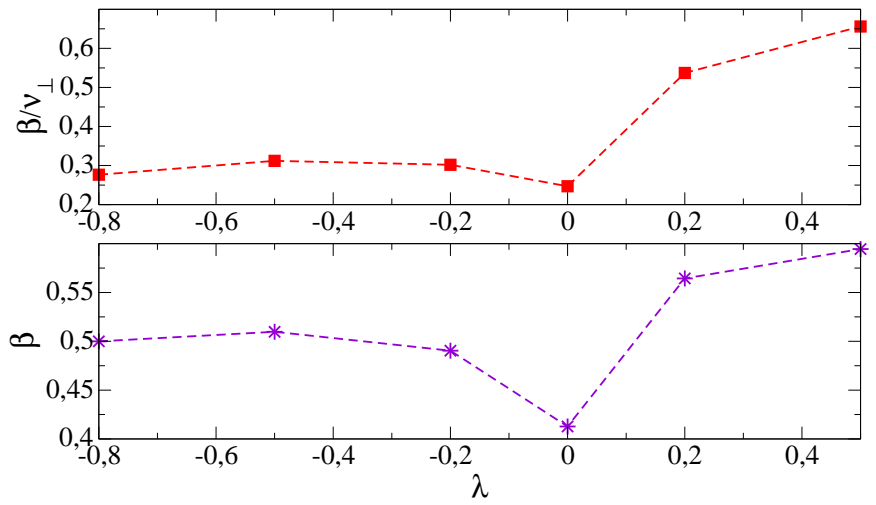

FIG. 8: Estimates for the critical exponents $\beta$ e $\beta / v_{\perp}$ as functions of the parameter $\lambda$.

\section{CONCLUSION}

We studied a generalization of the one-dimensional Manna model with height restrictions and conservation of the number

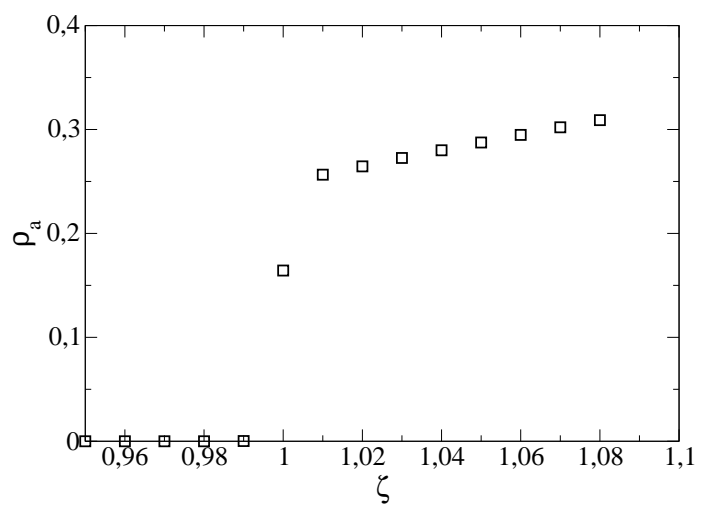

FIG. 9: Estimates for the order parameter for $L=500$, with $\lambda=1$, indicating a first order transition.

of particles, with the inclusion of a parameter $\lambda$ which is related to the two toppling processes which occur in the model. When $\lambda=-1$ no transition is found between an active and an absorbing state, and a diffusive dynamics of active sites is found. In the other extreme $\lambda=1$ the model corresponds to a conservative BTW sandpile, and our results indicate a discontinuous transition as obtained by Dickman et al in [7]. We believe that the observed variations in the critical exponents may be due to crossover effects in the two limiting cases of the model.

It is necessary to extend our simulations to values of $\lambda$ which are closer to both limits, in order to find out if the exponents approach limiting values. In particular, it would be interesting to find out if mean-field exponents are found as $\lambda \rightarrow-1$. The exponent ration $v_{\|} / v_{\perp}$ should also be estimated through additional simulations, as well as the ratio $m=\left\langle\rho_{a}^{2}\right\rangle / \rho_{a}^{2}$, whose value at $\zeta=\zeta_{c}$ is also universal. We believe the phase transition in the model to be discontinuous only at $\lambda=1$, but the data we have collected so far do not allow us to discard the possibility that a tricritical point exists for some value of $\lambda$ between 0 and 1 .

We are presently trying to answer these questions, so that the critical behavior of this model, which does not belong to the DP universality class, may be better known.

\section{Acknowledgments}

We thank Prof. Ronald Dickman for many helpful discussions. This research was partially supported by the Brazilian agencies CAPES, FAPERJ and CNPq, whose assistance is gratefully acknowledged. An author, J.F. Stilck, acknowledges support through project PRONEXCNPq-FAPERJ/171.168-2003. 
[1] H. Hinrichsen, Adv. Phys.49, 815 (2000).

[2] J. Marro and R. Dickman, Nonequilibrium Phase Transitions in Lattice Models (Cambridge University Press, Cambridge, 1999).

[3] T. E. Harris, Ann. Probab. 2, 969 (1974).

[4] H. K. Janssen, Z. Phys. B 42, 151 (1981); P. Grassberger, Z. Phys. B 47, 465 (1982).

[5] I. Jensen, Phys. Rev. Lett. 70, 1465 (1993).

[6] R. Dickman and I. Jensen, Phys. Rev. E 48, 1710 (1993).

[7] R. Dickman et al., Phys. Rev. E 64, 056104 (2001).

[8] R. Dickman, T. Tomé, and M. J. de Oliveira, Phys. Rev. E 66
016111 (2002).

[9] S. S. Manna, J. Stat. Phys. 59, 509 (1990); J. Phys. A 24, L363 (1991).

[10] A. Vespignani and S. Zapperi, Phys. Rev. Lett. 78, 4793 (1997); R. Dickman, A. Vespignani, and S. Zapperi, Phys. Rev. E 57, 5095 (1998).

[11] P. Bak, C. Tang, and K. Wiesenfeld, Phys. Rev. Lett. 59, 381 (1987); Phys. Rev. A 38, 364 (1988).

[12] M. M. de Oliveira and R. Dickman, Phys. Rev. E 71, 016129 (2005). 